\title{
Insomnie bei Demenzen
}

\author{
Sleep disorders and dementia
}

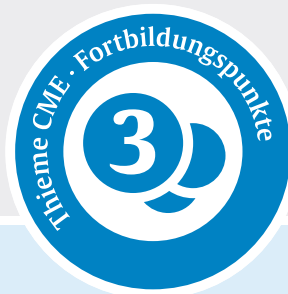

Geert Mayer ${ }^{1}$, Helmut Frohnhofen ${ }^{2}$

1 Abteilung für Neurologie, Philipps-Universität Marburg

2 Fakultät für Gesundheit, Department Humanmedizin, Universität Witten-Herdecke

\section{ZUSAMMENFASSUNG}

Schlafstörungen gehen Demenzen häufig voran und können deren Verlauf verschlimmern. Die Interaktion ist bidirektional. Beide Erkrankungen haben gemeinsame Symptome wie kognitive Einschränkungen und Veränderungen von Schlafstruktur und Qualität. Wenn sich die Demenz einmal manifestiert hat, können Schlafstörungen wie obstruktive Schlafapnoe, Restless-legs-Syndrom und Parasomnien die Symptomatik verstärken. Die Schlafstruktur zeigt eine Verminderung von langsamwelligem Schlaf und häufiges nächtliches Erwachen mit längeren Wachzeiten. Schlaf ermöglicht es, mittels Aktivierung des glymphatischen Systems Aß- und Tau-Proteine, die sich im Wachen angesammelt haben, aus dem ZNS aus- zuschleusen. Wegen der bidirektionalen Interaktionen ist es erforderlich, Schlafstörungen vermehrte Aufmerksamkeit zu schenken und sie früh zu diagnostizieren und zu behandeln, um einer Verschlimmerung einer existierenden oder sich entwickelnden Demenz vorzubeugen. Eine Anzahl von subjektiven Fragebögen und objektiven Instrumenten wie Polysomnografie und Aktigrafie stehen für die Diagnostik zur Verfügung. Begleitende Schlafstörungen sollten immer mit behandelt werden (z. B. nasale Überdrucktherapie bei Schlafapnoe).

Prävention oder Behandlung sollten immer Verhaltenstherapien wie körperliche Aktivierung, soziale Interaktion und ausreichende Exposition gegenüber Tageslicht beinhalten. Einige Antidepressiva, Melatonin, neue duale Orexinrezeptorantagonisten (in Deutschland nicht zugelassen) und wenige Hypnotika können zur Behandlung empfohlen werden. Die Schlafprobleme der betreuenden Personen sollten in den Behandlungsablauf eingeschlossen werden, um die Lebensqualität der Erkrankten zu optimieren.

\section{Einleitung}

\section{Ca. 25\%-50\% aller Patienten mit Alzheimer-Demenz oder} anderen Demenzformen leiden unter Schlafstörungen. Die Schlafforschung konnte zeigen, dass Schlafstörungen das Risiko eine Demenz zu entwickeln erhöhen, den Verlauf einer Demenz verschlimmern können, einige ähnliche Symptome haben und ein früher Demenzmarker sein können. Es ist daher wichtig, bei Schlafstörungen frühzeitig nach Anzeichen von Demenzen zu fragen und andererseits bei Demenzen und milden kognitiven Störungen nach Schlafstörungen zu fragen. Die frühe Diagnose von Schlafstörungen bei Risikopatienten kann zu einer frühen Prävention und kausalen Therapie beitragen und den Krankheitsverlauf entscheidend beeinflussen. Der Schwerpunkt dieses Beitrags liegt in der Diagnostik und Therapie von Insomnien bei Demenzen.

Insomnien werden gemäß der Internationalen Klassifikation der Schlafstörungen (American Academy of Sleep Medicine ICSD3 2014) [1] in akute (<3 Monate Dauer) und chronische (>3Monate Dauer) Formen eingeteilt. Bei älteren Menschen ändert sich das Schlafbedürfnis im Vergleich zu jüngeren Menschen nur wenig, allerdings ist die Fähigkeit, ausreichende Mengen einzelner Schlafstadien zu bekommen, gegenüber Jüngeren vermindert und die zirkadiane Verteilung ist verändert [26]. Die häufigsten komorbiden Schlafstörungen sind die Schlafapnoen, Restless-legs-Syn- drom und REM-Schlafverhaltensstörung. Schlafstörungen können die Entstehung von Demenzen begünstigen und Demenzen ihrerseits Schlafstörungen verursachen. Insomnien erhöhen das Risiko einer kognitiven Verschlechterung um 27 \% [35]. Über 50-65 Jahre alte Patienten mit einer lang bestehenden Insomnie haben ein höheres Demenzrisiko als über 65-Jährige ohne Insomnie. Gebrauch von hochdosierten Hypnotika mit einer langen Halbwertzeit erhöhen ebenfalls das Demenzrisiko [7]. 29\% von über 55-jährigen mit Mild Cognitive Impairment (MCI) haben in prospektiven Studien eine Alzheimer-Demenz (AD) entwickelt. Das Risiko hierfür vermindert sich durch komorbide affektive Störungen [28]. Insomnische Symptome sind bei Alzheimer-Demenz-Patienten mit Störungen der frontalen exekutiv- und visuospatiale Funktionen assoziiert [31].

\section{Pathophysiologie der Insomnie bei Demenzen}

Schlafstörungen können neurodegenerative Prozesse durch vermehrte Produktion oder Ablagerung und verminderte glymphatische Clearance von ß-Amyloid, Tau-Protein und alpha-Synuclein fördern. Störungen des zirkadianen Rhythmus (intrinsisch und verhaltensbedingt), Schlaffragmentierung, entzündliche ZNS-Prozesse und oxidativer Stress können zu synaptischen Störungen und Neurodegeneration beitragen. Die neuronalen Schädi- 
gungen können unabhängig vom Schädigungstyp unterschiedliche Schlafstörungen hervorrufen, weshalb mehrere Formen von Schlafstörungen bei einer Person auftreten können und im Verlauf des neurodegenerativen Prozesses entstehen können. Das Risiko eine Demenz zu entwickeln ist abhängig von genetischen Faktoren (apo-E4) und vom individuellen Lebensstil [17]. Insomnien tragen durch die Schlaffragmentierung in besonderer Weise zur Entstehung der Alzheimer-Demenz bei. Das glymphatische System scheidet im Schlaf „Abfallprodukte“ wie z. B. fehlgefaltete Proteine, die sich im Wachen im Gehirn ansammeln, aus. Durch Insomnien verringert sich die Dauer der Aß-Ausscheidung über das glymphatische System und führt zu einer Akkumulation im Gehirn. Die Aggregation von Beta-Amyloid (Aß) kann schon 20 Jahre vor Manifestation einer Demenz auftreten und eine Verminderung von REM- und Tiefschlaf sind schon vorhanden [18]. Schlafbezogene Atmungsstörungen zeigen ein erhöhtes t-tau/Aß42-Verhältnis und können durch apnoebedingte Schlaffragmentierung und repetitive apnoeassoziierte Hypoxämien in der Nacht zusätzlich hirnschädigend wirken. Diese Veränderungen verstärken altersbedingte degenerative Veränderungen von Neurotransmittern und zirkadianen Rhythmen. Im Alter werden der zirkadiane Rhythmus in der Amplitude und die nächtliche Melatoninkonzentration [32] gedämpft. Durch Veränderung externer Faktoren (Zeitgeber) wie z. B. mangelnde Blaulichtexposition, Verlust von melanopsinhaltigen retinalen Ganglionzellen durch Amyloidablagerungen in der Retina und verminderter körperlicher Aktivität werden die zirkadianen Rhythmen weiter vermindert.

\section{Schlafstörungen bei unterschiedlichen Demenzformen}

Alzheimer-Demenz (AD): Die Häufigkeit von Schlafstörungen liegt zwischen 30\%-60\%. Der Schlaf ist fragmentiert, die Schlafeffizienz in der Nacht verkürzt, das Schlafstadium N2 zeigt vermindertes Auftreten von Schlafspindeln und K-Komplexen, die für die Kognition von Bedeutung sind, der Tiefschlafanteil in der Nacht ist vermindert.

Vaskuläre Demenz: 80 \% aller Patienten leiden unter Schlafstörungen, bei $70 \%$ bestehen eine Schlafapnoe, Insomnie bei $67 \%$, REM-Schlafverhaltensstörung bei $25 \%$ und RLS bei $5 \%$ [11].

Frontotemporale Demenz (FTD): Ca. 70 \% aller Patienten mit früher FTD haben Schlafstörungen. Die Patienten haben oft einen vorverschobenen zirkadianen Rhythmus, Schlafapnoe tritt bei etwa $68 \%$, exzessive Tagesschläfrigkeit bei $64 \%$, Insomnie bei $48 \%$ und Restless-legs-Syndrom bei $8 \%$ auf [11].

Demenz vom Lewy-Körper-Typ (LBD): Die Prävalenz von Schlafstörungen liegt bei fast $90 \%$ aller Patienten mit LBD. Schlafbezogene Atmungsstörungen sind mit 76\% am häu- figsten, gefolgt von exzessiver Tagesschläfigkeit (71\%), Insomnie (67\%), REM-Schlafverhaltensstörung (48\%). Die REM-Schlafstörung und die Tagesschläfrigkeit sind gemäß den „Revised criteria for the clinical diagnosis of probable and possible LBD” [23] klinische Kernsymptome.

Creutzfeldt-Jakob-Erkrankung: Zahlen zur Prävalenz von den häufig auftretenden Schlafstörungen sind nicht vorhanden. Der Verlauf kann von der Behandlung der komorbiden Schlafstörungen profitieren [13].

\section{Diagnostik}

Die Erhebung der Anamnese ist abhängig von den kognitiven Möglichkeiten der Betroffenen bzw. ihrer Fähigkeit, ausreichend Auskunft über ihre Krankengeschichte zu erteilen. Im Falle einer eingeschränkten Verständigung muss eine Anamnese durch die Familienangehörigen oder Betreuer erfolgen. Diese sollte die Schlafgewohnheiten und -probleme, Verhaltensprobleme, die medizinische Anamnese, die derzeitige Medikation und ggfs. Substanzen einschließen. Die tageszeitliche Gabe der Medikation ist detailliert zu erheben, um z. B. die abendliche Gabe eines Diuretikums, das zu Nykturie führen kann, zu ändern. Lebens- und Wohnsituation, Art und Häufigkeit körperlicher Aktivitäten, Lichtexposition am Tag und in der Nacht, Tagesstruktur und weitere kontribuierende Faktoren wie das Vorhandensein von Depressionen und Angststörungen müssen berücksichtigt werden.

Schlaftagebücher - geführt von den Patienten oder Versorgern - können helfen die Ursachen der Schlafprobleme zu erfassen. Schlafstörungen bei dementen Patienten können mittels Fragebögen [5] erfasst werden, Insomnien durch den Pittsburgh Sleep Quality Index [6], deren Schweregrad durch die Insomnia Severity Scale, Tagesschläfrigkeit durch die Epworth Sleepiness Scale (ESS) [12]. Spezifische, validierte Insomniefragebögen für demente Patienten gibt es nicht. Mittels Aktigrafie können SchlafWach-Zeiten, nächtliches Erwachen, Tagschlafepisoden und motorische Aktivität, ggfs. auch bei integriertem Photometer die tägliche Lichtmenge gespeichert werden [2]. Das Steering Committee of the American Academy of Sleep Medicine hat Schlaftagebücher zur Diagnostik von irregulären Schlaf-Wach-Mustern bei Demenzen und Restless legs empfohlen [25]. Eine Untersuchung im Schlaflabor ist nur bei komplexer Fragestellung erforderlich (z. B. Abgrenzung einer REM-Schlafverhaltensstörung von einer nächtlichen Epilepsie), wenn die aus den Ergebnissen resultierenden Konsequenzen umsetzbar erscheinen.

\section{Therapien}

Zur Übersicht von Therapien sei auf Übersichten verwiesen [19]. Eine optimale Schlafhygiene sollte immer umgesetzt werden, auch wenn hierzu kaum evidenzbasierte Daten vorliegen [21]. Bei milden Formen der Demenz kann eine 
kognitive Verhaltenstherapie der Insomnie erprobt werden. Eine Kombination von Laufen und Echtlichtexposition (2500 Lux) von > 4 Tagen/Woche über 6 Monate verbesserte bei Patienten mit AD ihre aktigrafisch gemessene Schlafdauer [22]. Melatonin (bis $10 \mathrm{mg}$ unretardiert und 2,5 mg retardiert) hatte keinen Effekt auf Schlafstörungen bei Alzheimer-Demenz [20]. Die Leitlinie der Amerikanischen Akademie für Schlafstörungen [1, 3] empfiehlt die Behandlung von Melatonin bei Demenzen unter Berücksichtigung des positiven Effekts auf die Schlaf-Wach-Rhythmusstörungen. Bei komorbider REM-Schlafverhaltensstörung ist $2,5 \mathrm{mg}$ Melatonin in retardierter Form immer zum gleichen Zeitpunkt gegeben indiziert [15].

Trazodon 50 mg zeigte eine Verlängerung der Schlafdauer und Schlafeffizienz, aber keinerlei Effekt auf die nächtliche Wachzeit und die Häufigkeit des Erwachens. Eine placebokontrollierte Studie mit $15 \mathrm{mg}$ Mirtazapin über 2 Wochen um 21 Uhr verabreicht, zeigte bei aktigrafischer Schlafüberprüfung keinen schlaffördernden Effekt, allerdings war die Studie mit 8 behandelten Patienten und 18 Kontrollen sehr klein [33]. Orexinagonisten können in der Zukunft eine Rolle bei der Behandlung der Insomnien spielen, sie sind jedoch noch nicht bei Demenzpatienten eingesetzt worden [30]. Bei komorbider behandlungsbedürftiger Schlafapnoe kann eine CPAP-Therapie erprobt werden, deren Erfolg von der Toleranz und Adhärenz abhängig ist [8].

Demenzpatienten werden häufig mit Neuroleptika behandelt. Eine Demenz-Leitlinie empfiehlt, bei Erwachsenen mit primärer Insomnie und bei sekundären Insomnien mit behandelten Komorbiditäten Neuroleptika abzusetzen [4], allerdings zeigt eine Cochrane-Metaanalyse wenig oder keine Evidenz für einen Effekt von Neuroleptika auf die insomnischen Symptome [9]. Für die in der gängigen Praxis verordneten Hypnotika wie Benzodiazepine, Benzodiazepinrezeptoragonisten und Phytotherapeutika konnten keine Studien mit positivem Nutzen für Demenzkranke mit Insomnie gefunden werden. Diese Medikamente sollten primär nicht zur Insomniebehandlung verabreicht werden, da sie zu kognitiven Verschlechterungen, Stürzen und Tagesschläfrigkeit führen können, Depressionen und Angst verstärken können und den Schlaf nicht bessern [10]. Leider existiert keine Literatur über die Behandlung mit Cholinesteraseinhibitoren. Aus einer Studie bei älteren Patienten ist lediglich bekannt, dass sie durch das Auftreten von luziden Träumen irritierend wirken können [16]. Eine morgendliche Gabe könnte deren Auftreten vermutlich verhindern.
Interessenkonflikt

Erklärung zu finanziellen Interessen

Forschungsförderung erhalten: nein; Honorar/geldwerten Vorteil für Referententätigkeit erhalten: ja; Bezahlter Berater/interner Schulungsreferent/Gehaltsempfänger: ja; Patent/Geschäftsanteile/Aktien (Autor/Partner, Ehepartner, Kinder) an Firma (Nicht-Sponsor der Veranstaltung): nein; Patent/Geschäftsanteile/Aktien (Autor/Partner, Ehepartner, Kinder) an Firma (Sponsor der Veranstaltung): nein.

Erklärung zu nicht finanziellen Interessen

Schatzmeister der International REM Sleep Behavior Study Group

\section{Korrespondenzadresse}

Prof. Dr. med. Geert Mayer

Hephata Klinik

Schimmelpfengstr. 6

34613 Schwalmstadt, Deutschland

Tel. 06691/182002

geert.mayer@hephata.de

Literatur

[1] American Academy of Sleep Medicine. The International Classificatio of Sleep Disorders (3rd ed.). Darien IL: American Academy of Sleep Medicine, 2014

[2] Ancoli-Israel S, Cole R, Alessi C et al. The role of actigraphy in the study of sleep and circadian rhythms. Sleep 2003; 26 (3): 342-92. doi:10.1093/sleep/26.3.342

[3] Auger RR, Burgess HJ, Emens JS et al. Clinical practice guideline for the treatment of intrinsic circadian rhythm sleepwake disorders: advanced sleep-wake disorder (ASWPD), delayed sleep-wake phase disorder (DSWPD), non-24-hour sleep-wake rhythm disorder (N24SWD), and irregular sleepwake rhythm disorder (ISWRD). An update for 2015.J Clin Sleep Med 2015; 11: 1199-1236. doi: 10.5664/jcsm.5100

[4] Bjerre LM, Farrell B, Hogl M et al. Deprescribing antipsychotics for behavioural and psychological symptoms of dementia and insomnia. Canadian Family Physician 2018; 64: 17-27. PMID: 29358245; PMCID: PMC5962971

[5] Bloom HG, Ahmed I, Alessi CA et al. Evidence-based recommendations for the assessment and management of sleep disorders in older persons. J Am Geriatr Soc 2009; 57 (5): 761-89. doi: 10.1111/j.1532-5415.2009.02220.x

[6] Buysse DJ, Reynolds CF, Monk TH et al. The Pittsburgh Sleep Quality Index: a new instrument for psychiatric practice and research. Psychiatry Res 1989; 28 (2): 193-213. doi:10.1016/0165-1781(89)90047-4.PMID: 2748771

[7] Chen P-L, Lee W-J, Sun W-Z et al. Risk of Dementia in Patients with Insomnia and Long-term Use of Hypnotics: A Population-based Retrospective Cohort Study. PLoS ONE 2012; 7 (11): e49113. doi:10.1371/journal.pone.0049113

[8] Chong MS, Ayalon L, Marler M et al. Continuous positive airway pressure reduces subjective daytime sleepiness in patients with mild to moderate Alzheimer's disease with sleep disordered breathing. J Am Geriatr Soc 2006; 54 (5): 777-81. doi: 10.1111/j.1532-5415.2006.00694.x.PMID: 16696743 
[9] Declercq T, Petrovic M, Azermai M et al. Withdrawal versus continuation of chronic antipsychotic drugs for behavioural and psychological symptoms in older people with dementia. Cochrane Database Syst Rev 2013; (3): CD007726. doi:10.1002/14651858.CD007726.pub3

[10] Defrancesco M, Marksteiner J, Fleischhacker WW et al. Use of Benzodiazepines in Alzheimer's Disease: A Systematic Review of Literature. Int J Neuropsychopharmacol 2015; 18 (10): pyv055. doi:10.1093/ijnp/pyv055

[11] Guarnieri B, Adorni F, Musicco M et al. Prevalence of sleep disturbances in mild cognitive impairment and dementing disorders: a multicenter Italian clinical cross-sectional study on 431 patients. Dement Geriatr Cogn Disord 2012; 33 (1): 50-8. doi:10.1159/000335363

[12] Johns MW. Daytime sleepiness, snoring, and obstructive sleep apnea. The Epworth Sleepiness Scale. Chest 1993; 103 (1): 30-6. doi:10.1378/chest.103.1.30

[13] Kang P, de Bruin GS, Wang LH et al. Sleep pathology in Creutzfeldt-Jakob disease. J Clin Sleep Med 2016; 12 (7): 1033-1039. PMCID: PMC1232733

[14] Kemp S, Biswas R, Neumann $V$ et al. The value of melatonin for sleep disorders occurring post-head injury: a pilot RCT. Brain Inj 2004; 18(9): 911-919. doi:10.1080/02699050410 001671892

[15] Kunz D, Mahlberg R. A two-part, double-blind, placebocontrolled trial of exogenous melatonin in REM sleep behavior disorder. J Sleep Res 2010; 19: 591-596. doi:10.1111/ j.1365-2869.2010.00848.x

[16] LaBerge S, LaMarca K, Baird B. Pre-sleep treatment with galantamine stimulates lucid dreaming: A double-blind, placebo-controlled, crossover study. PLoS ONE 2018; 13 (8). doi:10.1371/journal.pone.0201246

[17] Livingston G, Sommerlad A, Orgeta V et al. Dementia prevention, intervention, and care. Lancet 2017; 390 (10113): 2673-734. doi:10.1016/S0140-6736(17)31363-6

[18] Mander BA, Winer JR, Walker MP. Sleep and Human Aging. Neuron 2017; 94(1): 19-36. doi:10.1016/j.neuron. 2017.02.004

[19] Mayer $G$ et al. Insomnie bei neurologischen Erkrankungen, S2kLeitlinie, 2020. In: Deutsche Gesellschaft für Neurologie (Hrsg.), Leitlinien für Diagnostik und Therapie in der Neurologie. www.dgn.org/leitlinien. doi:10.1186/s42466021-00106-3

[20] McCleery J, Cohen DA, Sharpley AL. Pharmacotherapies for sleep disturbances in dementia. Cochrane Database of Systematic Reviews 2016; 11. Art. No.: CD009178. doi:10.1002/14651858.CD009178.pub3

[21] McCurry SM, Gibbons LE, Logsdon RG et al. Nighttime insomnia treatment and education for Alzheimer's disease: a randomized, controlled trial. J Am Geriatr Soc 2005; 53 (5): 793-802. doi:10.1111/j.1532-5415.2005.53252.x

[22] McCurry SM, Pike KC, Vitiello MV et al. Increasing walking and bright light exposure to improve sleep in communitydwelling persons with Alzheimer's disease: results of a randomized, controlled trial. J Am Geriatr Soc 2011; 59 (8): 1393-1402. doi:10.1111/j.1532-5415.2011.03519.x

[23] McKeith IG, Boeve BF, Dickson DW et al. Diagnosis and management of dementia with Lewy bodies: Fourth consensus report of the DLB Consortium. Neurology 2017; 89 (1): 88-100. doi:10.1016/S1474-4422(19)30153-X

[24] Merlino G, Piani A, Gigli GL et al. Daytime sleepiness is associated with dementia and cognitive decline in older Italian adults: a population-based study. Sleep Med 2010; 11 (4): 372-7. doi:10.1016/j.sleep.2009.07.018
[25] Morgenthaler T, Alessi C, Friedman L et al. Practice parameters for the use of actigraphy in the assessment of sleep and sleep disorders: an update for 2007. Sleep 2007; 30 (4): 519-29. doi:10.1093/sleep/30.11.1445

[26] Ohayon MM. Sleep and the elderly. J Psychosom Res 2004; 56 (5): 463-4. doi:10.1016/j.jpsychores.2004.03.001

[27] Ouellet M-C, Morin CM. Efficacy of cognitive-behavioral therapy for insomnia associated with traumatic brain injury: a single-case experimental design. Arch Phys Med Rehabil 2007; 88 (12): 1581-1592. doi:10.1016/j. apmr.2007.09.006

[28] Ramakers IH, Visser P], Aalten P et al. Affective symptoms as predictors of Alzheimer's disease in subjects with mild cognitive impairment: a 10-year follow-up study. Psychol Med 2010; 40 (7): 1193-201. doi:10.1017/S0033291709991577

[29] Rongve A, Boeve BF, Arsland D. Frequency and correlates of caregiver-reported sleep disturbances in a sample of persons with early dementia. J Am Geriatr Soc 2010; 58 (3): 480-6. doi:10.1111/j.1532-5415.2010.02733.x

[30] Schroeck JL, Ford J, Conway EL et al. Review of Safety and Efficacy of Sleep Medicines in Older Adults. Clin Ther 2016; 38 (11): 2340-72. doi:10.1016/j.clinthera.2016.09.010

[31] Shin HY, Han HJ, Shin D] et al. Sleep problems associated with behavioral and psychological symptoms as well as cognitive functions in Alzheimer's disease. J Clin Neurol 2014; 10 (3): 203-9. doi:10.3988/jcn.2014.10.3.203

[32] Rodenbeck A, Hajak G. Neuroendocrine dysregulation in primary insomnia. Rev Neurol 2001; 155: 5S57-5S61. PMID: 11924040

[33] Scoralick FM, Louzada LL, Quintas JL et al. Mirtazapine does not improve sleep disorders in Alzheimer's disease: results from a double-blind, placebo-controlled pilot study. Psychogeriatrics 2017; 17 (2): 89-96. doi:10.1111/psyg.12191

[34] Theadom A, Cropley M, Parmar P et al. BIONIC Research Group. Sleep difficulties one year following mild traumatic brain injury in a population-based study. Sleep Med 2015; 16 (8): 926-932. doi:10.1016/j.sleep.2015.04.013

[35] Xu W, Tan C-C, Zou J-J et al. Sleep problems and risk of allcause cognitive decline or dementia: an updated systematic review and meta-analysis. J Neurol Neurosurg Psychiatry 2020; 91: 236-244. doi:10.1136/jnnp-2019-321896

Bibliografie

Nervenheilkunde 2021; 40: 511-514

DOI 10.1055/a-1466-9471

ISSN 0722-1541

(C) 2021. Thieme. All rights reserved.

Georg Thieme Verlag KG, Rüdigerstraße 14,

70469 Stuttgart, Germany 


\section{Punkte sammeln auf CM/F.thieme.de}

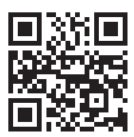

Diese Fortbildungseinheit ist bis zu 12 Monate online für die Teilnahme verfügbar.

Den genauen Einsendeschluss finden Sie beim Modul auf https://cme.thieme.de/CXH99W5.

Sollten Sie Fragen zur Online-Teilnahme haben, finden Sie unter https://cme.thieme.de/hilfe eine ausführliche Anleitung. Wir wünschen viel Erfolg beim Beantworten der Fragen!

Unter https://eref.thieme.de/CXH99W5 oder über den QR-Code kommen Sie direkt zum Artikel.

VNR 2760512021160211177

\section{Frage 1}

Was ist ist korrekt?

A Schlafstörungen erhöhen das Demenzrisiko nicht.

B Schlafstörungen erhöhen das Demenzrisiko nur bei schlafbezogenen Atmungsstörungen.

C Schlafstörungen können den Verlauf einer Demenz verschlimmern.

D Schlafstörungen haben keinen Einfluss auf den Verlauf von Demenzen.

E Demenzen haben keinen Einfluss auf Schlafstörungen.

\section{Frage 2}

Welche Antwort ist richtig? Personen > 55 Jahre mit MCI (mild cognitive impairment) ...

A entwickeln generell Demenzen.

B entwickeln generell Alzheimer-Demenz.

C haben ein geringeres Risiko eine Alzheimer-Demenz zu entwickeln bei komorbiden affektiven Störungen.

D haben ein stärkeres Risiko eine Alzheimer-Demenz zu entwickeln bei komorbiden affektiven Störungen.

E haben ein geringeres Risiko eine Alzheimer-Demenz zu entwickeln bei komorbiden schlafbezogenen Atmungsstörungen.

\section{Frage 3}

Das glymphatische System ..

A hat eine konstante 24-Stunden-Aktivität.

$B$ ist tagsüber aktiver als nachts.

$C$ ist nachts aktiver als tagsüber.

D ist in seiner Aktivität abhängig von der Schlaflatenz.

$\mathrm{E}$ ist in seiner Aktivität unabhängig von der Schlafdauer.

\section{Frage 4}

Bei Menschen > 65 Jahren ist die Melatoninausscheidung ...

A im REM-Schlaf verstärkt.

B im NREM-Schlaf verstärkt.

$C$ in der Amplitude nicht gemindert.

$D$ in der Phase verschoben.

E generell im Schlaf vermindert.

\section{Frage 5}

Schlafstörungen sind am häufigsten bei folgender Demenzform:
A Alzheimer-Demenz.
B Demenz von Lewy-Körper-Typ.
C Frontotemporaler Demenz.
D Vaskulärer Demenz.
E Creutzfeld-Jakob-Demenz.

\section{Frage 6}

Welche Angabe zur Anamnese von Patienten mit Demenz und Schlafstörungen trifft nicht zu? Die Anamnese sollte beinhalten:
A motorische Aktivitäten
B Lichtexposition am Tag und in der Nacht
C psychiatrische Komorbidität
D chirurgische Vorerkrankungen
E Ausmaß der Selbstversorgung

\section{Frage 7}

Ein spezifischer diagnostischer Fragebogen zur Erfassung von Schlafstörungen ist der ...
A geriatrischer Depressions-Score.
B SF-36.
C Pittsburgh Sleep Quality Index.
D BDI-II.
E Aktometrie.

\section{Frage 8}

Schlaflaboruntersuchungen bei Patienten mit Demenzen sollten nur durchgeführt werden bei ...
A schlafbezogenen Atmungsstörungen.
B Verdacht auf REM-Schlafverhaltensstörung und Epilepsien.
C Restless-legs-Syndrom.
D Enuresis nocturna.
E Verdacht auf Parkinson-Erkrankung. 


\section{Punkte sammeln auf CME.thieme.de}

Fortsetzung ...

\section{Frage 9}

Welche Verhaltensmaßnahmen sind bei Patienten mit Demenz und Schlafstörungen durchzuführen?
A Nur körperliche Aktivierung
B Körperliche Aktivierung und Echtlichtexposition
C Nur Lichtexposition am Morgen
D Nur Lichtexposition am Abend
E Lichtexposition in der Nacht

\section{Frage 10}

Die Gabe von Benzodiazepinen und Benzodiazepinrezeptoragonisten zur Verbesserung des Nachtschlafes wird bei Patienten mit Demenz nicht empfohlen, weil ...
A sie den Tiefschlaf nicht vermehren.
B sie die Gesamtschlafzeit nicht verbessern.
C sie zu Abhängigkeit führen können.
D sie Stürze und kognitive Störungen verursachen können.
E sie paradoxen Schlaf verursachen können. 